\title{
Bulky extramedullary plasmacytomata: rare mode of relapse in myelomatosis
}

\author{
S V DAVIES, B JONES, * C M STARKIE, * J A MURRAY \\ From the Departments of Haematology and *Histopathology, Selly Oak Hospital, Birmingham
}

SUMMARY In two cases of myelomatosis relapse was accompanied by the development of bulky extramedullary plasma cell tumours, which created serious management problems. In both cases the histology was of a poorly differentiated plasmacytoid tumour with frequent mitoses. In one case paraprotein had completely disappeared from the serum and urine after six months' treatment with melphalan alone but did not correlate with improved survival.

It is concluded that extramedullary spread of myelomatosis in the form of bulky tumour deposits is a rare and ominous mode of tumour progression or relapse. Rises in serum and urinary paraprotein concentrations are not the inevitable signs of relapse.

The prognosis of myelomatosis has improved little over the past two decades. ${ }^{1}$ Symptomatic benefit can now be achieved in a large proportion of cases, although a minority will die of progressive disease soon after diagnosis. The median duration of survival for all patients entering the Medical Research Council's IVth Myelomatosis Trial was 26 months. ${ }^{2}$ In patients who respond to treatment initially recurrence or "relapse" is inevitable and occurs between two and six years after remission has been achieved. This is usually evident by reappearance of diagnostic features, most notably rising concentrations of monoclonal paraprotein in serum and urine. Insidious deterioration with little or no response to treatment will follow in most cases.

Extramedullary plasmacytomata ${ }^{3}$ constitute about $10 \%$ of plasma cell dyscrasias and are generally seen in one of two clinical settings: as "primary" plasmacytomata, $80 \%$ of which occur in the upper air passages and oral cavity and which carry a high cure rate; and as plasma cell tumours accompanied by myelomatosis (sometimes called "secondary" tumours). Most of the latter are present at diagnosis of myelomatosis and their presence confers a poor prognosis. Rare case reports ${ }^{45}$ exist of plasmacytomata presenting late in the course of myelomatosis, usually in the context of rapidly progressing disease.

Two cases of myelomatosis in relapse are reported in which the late emergence of bulky extramedullary disease presented serious management problems. In one case life-threatening haemorrhage from gastric tumours heralded relapse and predated typical serological changes.

\section{Case reports}

CASE 1

A 63 year old quality control inspector presented in March 1983 with bone pain, a fractured clavicle, and pneumonia. Investigations established a diagnosis of IgG1 $\times$ myelomatosis: serum paraprotein concentration was $23.3 \mathrm{~g} / 1$, IgA $2.5 \mathrm{~g} / \mathrm{l}$, IgM $0.48 \mathrm{~g} / \mathrm{l}$; urinary free light chain was observed "in a very small quantity".

Four-day courses of melphalan $7 \mathrm{mg} / \mathrm{m}^{2}$ repeated three weekly were started ("M7" protocol Medical Research Council's Vth Myelomatosis Trial). Radiotherapy was given to clavicle and sacrum with symptomatic benefit. Paraprotein was absent from serum and urine after only six months' treatment and after 13 courses of melphalan treatment was stopped.

In December 1984 the patient sustained a pathological fracture of the right femoral neck: he had received single dose radiotherapy to a painful lytic lesion at that site two days previously. A Monk's prosthesis was inserted and he developed an iliofemoral deep vein thrombosis. Six months later the prosthesis was revised because of severe pain on weight-bearing and evidence of loosening. In view of further pain this prosthesis was replaced in February 1986, when a Charnley total hip replacement was performed.

Three weeks before the last operation routine investigations had shown recurrence of the same 
paraprotein (IgG1 к $3.8 \mathrm{~g} / \mathrm{l}$ ) with "detectable" (but unquantifiable) urinary free light chain but no rise in serum $\beta$-2-microglobulin. These findings were felt to be suggestive, but not diagnostic, of early relapse.

Two weeks after hip replacement the patient was admitted as an emergency with massive haematemesis, a three day history of melaena, and a two week history of progressive (symmetrical) thyroid enlargement. The prothrombin time was within the therapeutic range and haemorrhage continued despite correction of anticoagulation. Emergency endoscopy showed active bleeding from the stomach and at operation three "malignant" ulcers were present in the anterior wall, two having infiltrated the serosa. A subtotal gastrectomy with Roux-en-Y anastomosis was performed. The ulcers showed histological features of extramedullary plasmacytomata. A needle biopsy specimen of the thyroid mass showed infiltration with plasma cells. Wound dehiscence and septicaemia complicated the immediate postoperative period. Gastrointestinal bleeding persisted, acute renal failure supervened, and the patient died four weeks after emergency admission. A further large spherical and polypoidal plasmacytoma (fig 1) was found in the caecum at postmortem examination.

\section{CASE 2}

A 56 year old woman presented with weight loss and symptoms of anaemia in March 1981. Nine months previously she had sustained a Colles' fracture. Investigations showed a normochromic normocytic anaemia (haemoglobin $5 \cdot 1 \mathrm{~g} / \mathrm{dl}$, erythrocyte sedimentation rate $140 \mathrm{~mm} /$ first hour) and numerous plasma cells in the peripheral blood film. IgA $\kappa$ myelomatosis was diagnosed. Combination chemotherapy with melphalan, prednisolone, and vincristine was started and she achieved "plateau phase" (defined as six months' stable serum and urinary paraprotein in the absence of evidence of progressing disease) after 18 months' treatment. Treatment was continued for a further 12 months (until December 1983).

Rising serum paraprotein (with increasing $\beta-2$ -

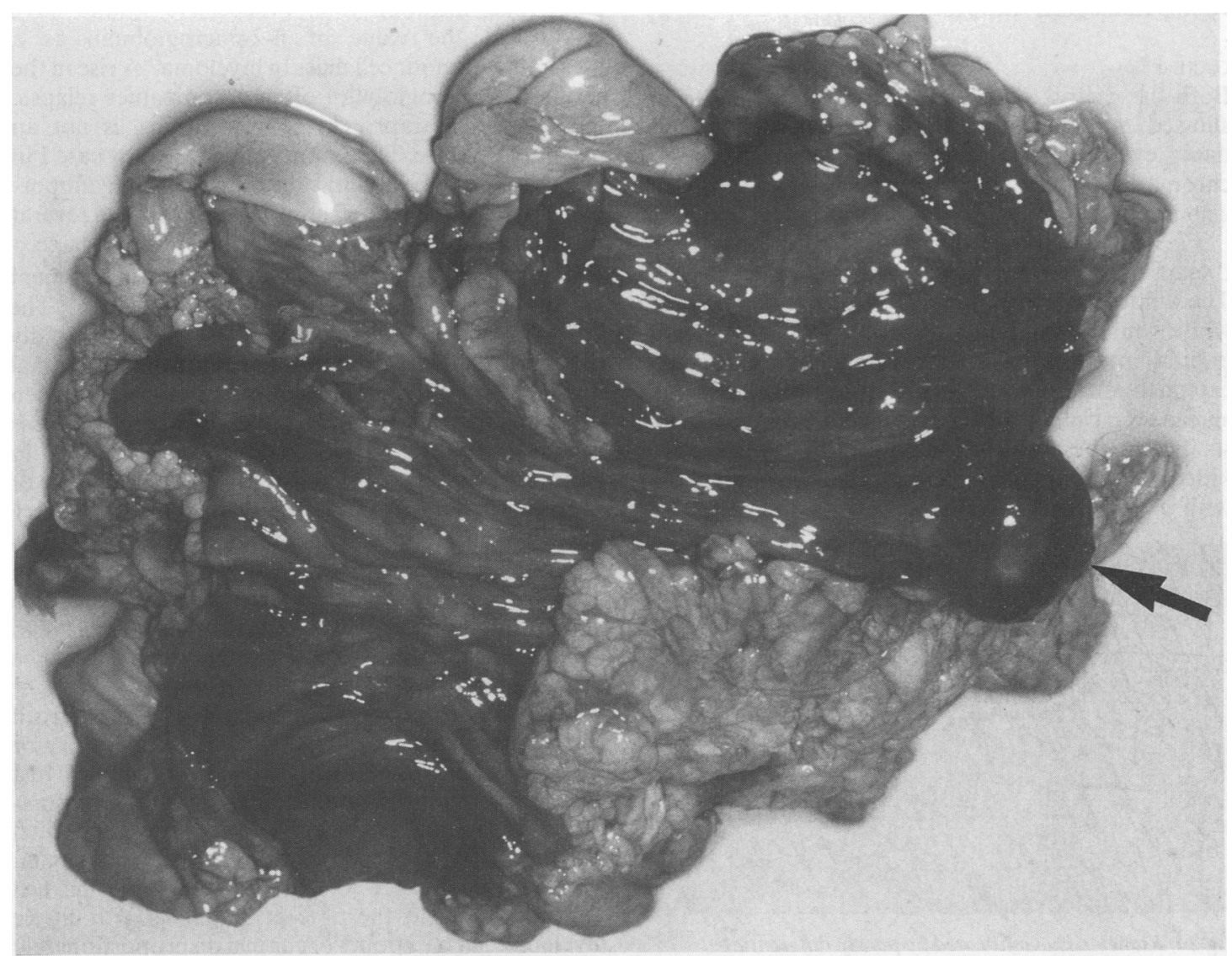

Fig 1 Large polypoidal plasmacytoma (arrowed) of caecum. 
microglobulin) led to reinstitution of melphalan in December 1984. Painful vertebral body collapse was treated with radiotherapy in June 1985. After transient serological response to melphalan weekly intravenous cyclophosphamide was introduced in August 1985. Troublesome cystitis did not respond to Uromitexan but some symptomatic benefit was gained by regular doses of nitrofurantoin. Paraprotein concentrations continued to rise despite cyclophosphamide, pulsed high dose prednisolone, and $\alpha$-2-interferon. In September 1986 she developed frank haematuria, cyclophosphamide having been stopped several months earlier. Cystoscopy showed a healthy bladder mucosa but an intravenous pyelogram and ultrasound examination showed a large right sided renal mass. Profuse haematuria requiring transfusion every few days persisted, and a nephrectomy was performed. An extensive tumour occupying the upper half of the kidney was found to be an extramedullary plasmacytoma (fig 2). She developed hypercalcaemia and renal failure and died four weeks later.

\section{Histopathological features}

\section{CASE 1}

Both the gastric ulcers and the lesion in the caecum showed immature plasma cell forms: most had abundant cytoplasm and a finely stippled nuclear chromatin pattern; multinucleated giant cells were also present.

\section{CASE 2}

The kidney measured $12 \times 6 \times 5 \mathrm{~cm}$ and showed a white rounded nodule $4 \times 3 \mathrm{~cm}$ replacing a renal papilla in the upper half of the kidney. Tumour extended into the renal pelvis but not the cortex. The surface was yellow and focally haemorrhagic. Microscopically, there was a pronounced infiltrate into the kidney of moderately pleomorphic basophilic cells with plentiful RNA and many mitoses. The tumour

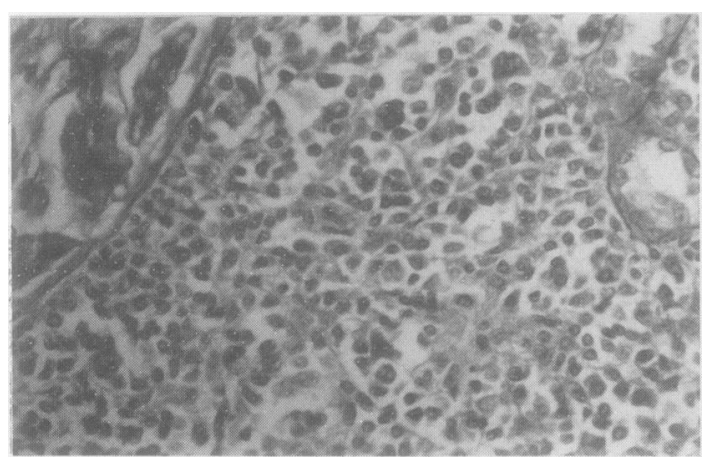

Fig 2 Kidney tissue infiltrated by poorly differentiated plasmacytoid cells. had the appearance of a poorly differentiated plasmacytoid lesion.

\section{Discussion}

The early detection of relapse and prompt reintroduction of treatment are of no established benefit in myelomatosis. Second responses to chemotherapeutic agents are at best occasional. ${ }^{1}$ Notwithstanding this, regular estimation of serum and urine paraprotein are in general use and a clinically important rise in either or both is considered to be an indication for further treatment. It is, however, well established that an emerging plasma cell clone may produce no detectable paraprotein, and in certain cases a fall in paraprotein accompanies relapse. ${ }^{3}$ Both situations reflect evolution of a less well differentiated tumour clone.

Beta-2-microglobulin is the light chain of the histocompatibility antigens found on the surface of most cells. Raised serum $\beta$-2-microglobulin concentrations have been recorded in a wide range of malignant diseases and are also associated with a reduction in glomerular filtration rate. Prospective studies have established the value of $\beta-2$-microglobulin as a measure of tumour cell mass in myeloma. ${ }^{6} \mathrm{~A}$ rise in the serum $\beta$-2-microglobulin often accompanies relapse, but, as with paraprotein concentrations, is not an inevitable feature. This is amply illustrated by case 1 in whom $\beta$-2-microglobulin showed no clinically important rise until acute renal failure had developed several weeks after the initial gastrointestinal haemorrhage.

The presentation of relapse due to the development of gross extramedullary disease is most unusual. ${ }^{45}$ The terminal phase of myeloma may exhibit a rapidly progressive illness with fever, pancytopenia, and renal impairment. A rare, more aggressive variant has been described in a small number of cases where relapse is accompanied by massive extramedullary disease. In two of these cases the histological impression of anaplasia was reinforced by DNA flow cytometry data: the tumour cell population showed hyperdiploidy and unusually high proliferative activity. In both the cases reported here the histology was of a poorly differentiated plasmacytoid tumour, and a high incidence of mitoses was notable. Our cases seem to be further examples of this unusual subtype.

Many reports exist of occasional cases of plasma cell tumours at disparate sites outside the respiratory or gastrointestinal tracts (reviewed by Bataille ${ }^{3}$ ). Most of these are descriptions of "primary plasmacytomata"- that is, plasma cell tumours which develop in the absence of any features of myeloma. The remainder refer to extramedullary plasma cell tumours found at the time of diagnosis of multiple myeloma. Such tumours occur in a disproportionately large number of cases of IgD myeloma, ${ }^{7}$ but no other 
association with paraprotein immunoglobulin type or subtype has been recognised. Reports of bulky extramedullary disease arising in the course of myeloma are extremely rare. Widespread extramedullary deposits can be found in as many as $70 \%$ of cases of myeloma at necropsy, ${ }^{8}$ but these are usually microscopic foci which would not be clinically detectable. The evolution of large solid tumours in the cases described here and elsewhere leads to "solid tumour" problems: haemorrhage, visceral obstruction, and local tissue destruction, which are not otherwise seen in myelomatosis.

Extramedullary plasmacytomata which are not associated with bone marrow disease carry an excellent prognosis and are usually cured by local treatment. This is particularly so for the $80 \%$ confined to the oral cavity and upper respiratory tract. ${ }^{3}$ Few cases relapse, and progression to multiple myeloma is rare. In contrast, the appearance of plasmacytomata in the course of myelomatosis carries an extremely poor prognosis. The limited reported experience suggests that the outlook is worse still when plasmacytomata arise in the context of myelomatous relapse.

Reference has been made to the use of local measures in the treatment of "primary" plasmacytomata, and surgery and radiotherapy may also have a role in the palliation of "solid tumour" symptoms in relapse with bulky disease. This was illustrated by the need for surgery in both cases described here. If any worthwhile impact on such disease is to be attempted, however, highly aggressive measures, such as multidrug intensive chemotherapy or double hemi-body irradiation, must also be taken.

Notably, in case 1, complete disappearance of paraprotein from serum and urine had been achieved after six months' treatment with melphalan alone. Such a rapid response carries a worse prognosis than a slower response, regardless of the chemotherapy regimen. ${ }^{910}$ Cases with complete disappearance of paraprotein are less likely to relapse in plateau phase when treatment stops than if maintenance treatment is given, but complete disappearance of paraprotein itself has not been correlated with improved survival.

In conclusion, extramedullary spread of myelomatosis in the form of bulky tumour deposits, as in the cases reported, constitutes a rare and ominous mode of tumour progression or relapse. Rapidly expanding friable extramedullary tumours may present serious and urgent management problems. Rises in serum and urinary paraprotein and in serum $\beta$-2-microglobulin are not the inevitable signs of relapse.

Grateful thanks are given to the medical illustration department for providing the photographs, and $\mathrm{Mr}$ Duncan Campbell and $\mathrm{Mr}$ Clive Young for their surgical expertise.

\section{References}

1 Durie BGM, Salmon SE. The current status and future prospects of treatment for multiple myeloma. Clin Haematol 1982;11: 181-210.

2 Medical Research Council Working Party on Leukaemia in Adults. Objective evaluation of the role of vincristine in induction and maintenance therapy for myelomatosis. $\mathrm{Br} J$ Cancer 1985;52:153-8.

3 Bataille R. Localised plasmacytomas. Clin Haematol 1982;11: 113-22.

4 Suchman AL, Coleman M, Mouradian JA, Wolf DJ, Saletan S. Aggressive plasma cell myeloma. Arch Intern Med 1981; 141:1315-20.

5 Foucar K, Raber M, Foucar E, Barlogie B, Sandler CM, Alexanian R. Anaplastic myeloma with massive extramedullary involvement. Cancer 1983;51:166-74.

6 Bataille R, Grenier J, Sany J. Beta-2-microglobulin in myeloma: optimal use for staging prognosis and treatment - a prospective study of 160 patients. Blood 1984;63:468.

7 Jancelewitz Z, Takatsuki K, Susai S. IgD multiple myeloma. Review of 133 cases. Arch Intern Med 1975;135:87.

8 Hayes DW, Bennett WA, Heck FJ. Extramedullary lesions in multiple myeloma. Review of literature and pathological studies. Archives of Pathology 1952;53:262-72.

9 Durie BGM, Salmon SE, Moon TE. Pretreatment tumour mass, cell kinetics, and prognosis in multiple myeloma. Blood 1980;55:364-72.

10 MacLennan ICM, Kelly K, Crockson RA, Cooper EH, Cuzick J, Chapman C. Results of the MRC myelomatosis trials for patients entered since 1980. Haematol Oncol 1988;6:145-58.

Requests for reprints to: Dr J A Murray, Department of Haematology, Queen Elizabeth Hospital, Queen Elizabeth Medical Centre, Edgbaston, Birmingham B15 2TH, England. 